\title{
Chinese Shuyuan: A Legacy in Chinese Education History, or a Solution for Modern Undergraduates in China?
}

\author{
Zhen Zeng $^{1}$ \\ ${ }^{1}$ School of Foreign Studies, Guangxi Normal University, Guilin, Guangxi, China \\ Correspondence: Zhen Zeng, 82 Liuhe Road, Qixing District, Guilin, Guangxi, Postal Code: 541004, China. \\ E-mail: joyzeng@163.com
}

Received: September 28, 2020 Accepted: November 19, 2020 Online Published: November 26, 2020

doi:10.5539/jel.v9n6p173 URL: https://doi.org/10.5539/jel.v9n6p173

\begin{abstract}
The paper looked into concepts claimed to be essence of Chinese residential college, an on-going institution presumed to be a solution towards undergraduates' issues in some pioneer universities in China. It's analyzed that Chinese residential college today in China is not a Shuyuan that was ever striving as a unique education mode in ancient China, even if it's named after Shuyuan in Chinese, concerning on its nature, function and goal, while it's not a conventional residential college in English speaking countries neither. By investigation and comparison of its origin, function and features among Shuyuan and Chinese residential college, the spirit of development of a human with goodness and well-being through pursuit of knowledge and culture inherited and transmitted in Shuyuan is unearthed, which is supposed to be the resource of inspiration when the pioneer universities and educators designed and operate residential college on Chinese campus, though the effects couldn't be accounted as appealing as what Shuyuan produced in ancient China. The research aims to depict a real Shuyuan, an education legacy every being alive in Chinese history, which were featured with paramount concepts and values that should be correctly recognized and implemented when there is great concern around undergraduates.
\end{abstract}

Keywords: shuyuan, Chinese residential college, undergraduate, comparison

\section{Introduction}

Due to intensified communication and exchange between Chinese and western countries and the further globalization assisting by technology initiation and creation in an incredible speed, Chinese higher education actively gets involved into this huge transforming movement in all aspects. Various concepts and diversified visions coming up and down in seconds, quickly disseminate every corner of China. On the one hand, these initiatives and concepts pouring from western culture and society enrich and broaden university students' visions and help them adapt to the world shared by people with multi-cultures and diversified life-style. On the other hand, such diversified visions are producing conflicts among Chinese inherent concepts and values widely. For example, utilitarianism, a concept prevailing in industrialized era supporting immediate profit and personal interests has progressively been penetrating among young people, leading to prejudice on their decision of choosing academic programs in university. Many students are turning into programs of science and technology rather than humanities programs, because Bachelor of Science would possibly produce immediate profit than Bachelor of Art when undergraduates enter their careers. It's a concerned fashion among stakeholders of Chinese higher education that undergraduates weigh technology over humanities, knowledge over intelligence, materialism over wisdom. Lu, Fang, then Vice President of Fudan University claimed, "professionally orientated education that focus on professional skills and technique would constrain learners into a very specific area-specific skill mastery" (Zhang, 2011). Against the convention of Chinese culture, it's well assumed that utilitarianism, a robust concept in China under current developmental stage, is a detriment towards morality, humanity and lofty spirit born with Chinese; overwhelming praise on professionally knowledge leads to ignorance of nourishing morality and humane value. "Undergraduates are lacking social responsibilities and commitment for social development addition to ignore the significance of humanity and humane values", Professor Lu said (Zhang, 2011). Fudan University is one of prestigious universities of the state. Concerning on undergraduates issues on the current context, Fudan Universtiy launched its reform towards undergraduates in 2006, establishing its own liberal education institution first, then, opening 5 Chinese residential colleges 
continuously that are administered by its Fudan College. Newly Fudan College is charging of undergraduates' issues, academically, ideologically, mentally, as well as physically. These residential colleges are all name after Shuyuan in its Chinese expressions. Except Fudan University and its Fudan College, several other universities, HongKong Chinese University, Xi'an Jiaotong University, Macau University, Southern University of Science and Technology launched their 'Shuyuan (书院)' projects and built up physical complexes. Under the pressure of higher education and concerns on undergraduates in the current situation of China, in order to assist undergraduates to adapt to the world successfully, Shuyuan (书院) with establishment of residential college in China, presumed a solution, comes back to vision of pioneer educators and universities highly developed.

However, many years operations (Hongkong Chinese Universtiy has been operating its residential college more than half a century) demonstrate that 'Shuyuan (书院)' project, residential colleges (Note 1) or residential hall in fact, hasn't been an accepted education concept across the country. There are few new partners coming in, enlarging the concept constructing and implementing around China, though those very first pioneer universities (like HongKong Chinese University, Fudan University, ect.) still keep on operating Chinese residential colleges and considering the project its specialty on campus. Considered the project's operation and the urgent demands of assisting undergraduates to develop successfully with globalization inclination, it's inquired that: why is Shuyuan the concept inspiring those pioneer projects towards undergraduates if Shuyuan (书院) was not an effective education mode? Why hasn't residential college, inspired by concept of Shuyuan, been popularized and adapted to more campus across the state if Chinese residential college has been effecting well in those reputational universities? and one more but not last inquiry is why is residential college in China translated into Shuyuan (书院) if it's not a practical concept being able to work in today Chinese higher education institutions?

On these questions, addition to the current situation confronting towards undergraduates, it's better to look into Shuyuan, its features, functions that truly inspired those pioneer 'Shuyuan' projects in Fudan University, HongKong Chinese University and those universities provoked by Shuyuan and establish Chinese residential colleges on their campus, with the purpose of interpretation of inquiries above.

\section{Researching Questions and Methods}

The paper is going to look into the following questions:

What is Shuyuan (书院), its attributes, features and operation that was once prosperous in Chinese history?

What are differences between Shuyuan (书院), Chinese residential college (Shuyuan(书院) in Chinese), and residential college in Western universities as well?

What are the inspiring essences of Shuyuan that undergraduates today in China would be mostly beneficial?

For interpretation and illustration of the matter, it's supposed to go over these questions and inquiries through methods of literature review, historical investigation and comparison to look into as much facts and historical events as possible for clarify the values of Shuyuan in ancient China and today as well.

\section{Ancient Chinese Shuyuan: Its Originality, Attributes, and Features}

\subsection{Original Story of Chinese Shuyuan}

Chinese Shuyuan (书院) was a quite peculiar education institution in Chinese education system. It's formulated from official institutions of Tang Dynasty (618-907), where were dedicated for books edited and books stored. LiZheng Shuyuan (立正书院) was an influential one then, built in 723 B.C. (the 11th year of KaiYuan, Tang Dynasty, when Emperor XuanZong was on the throne). The officials of the LiZheng Shuyuan were responsible for scholarly managing classic books edited in the past and uncovered at present in order to intelligently collect the policies and rules ever proposed and launched for governing a country because these officials were required to response the enquiries about ruling the country from the rulers all the time. In other words, officials working in Shuyuan were consultants or learning accompanies to the rulers on the throne, assisting them to master all knowledge for being an intelligent ruler. Gradually, high ranking officials and outstanding master scholars imitated to build 'Shuyuan (书院)' at home functioning LiZheng Shuyuan run by royal chamber, storing books and offering preschool education to their descendants. Such private learning place was turned into a private institution when official education institutions crashed due to occasionally social chaos caused by politics uncertainty in Chinese historic stages.

Under period of social uncertainties, for avoiding detriments, reputational master scholars lectured in countryside or somewhere apart from state court and political hubs as well, where they're assumed to be able to get away from political oppression and persecution while keep on freely pursuing knowledge. Moreover, many masters expected to, through transmitting their scholarly thoughts and doing researching in a remote area, relieve social 
conflicts and rejuvenate academic freedom pursued and prospered by master scholars of Confucius before Qin Dynasty (Paleolithic age-221 B.C.). Finally, such movement progressed into an influentially educational institution-Shuyuan that opened for all who would like to devote themselves into learning and researching with its very features from a typically private learning room.

Huang Yizhou (1894), a scholar in Qing Dynasty depicted the origin of Shuyuan (书院), “Lecturing was a very popular activity since Nan Song Dynasty (1127 A.D.-1279 A.D.). Once a knowledgeable lecturer died, his learners, estimated hundreds more, didn't dismiss immediately, but continued to discuss and explore the lecturer's views together around the lecturing place. Learners named the lecturer's house Chinese Shuyuan (书院) when excellent lecturing places were located at White Deer and Shigu evolving into White Deer Cave Shuyuan (白鹿洞书院, Note 2) and Shigu Shuyuan (石鼓书院)” (Huang, 1894).

In short, Chinese Shuyuan, basing on conditions mentioned above, was originated from an official institution appearing in Tang Dynasty and boomed in Song Dynasty (960 A.D.-1279 A.D.). It's developed into a systematically formulated educational institution from a private learning room due to historical needs.

\subsection{Nature of Shuyuan}

In Chinese education history, Shuyuan was neither an official education institution, nor private school. Chinese Shuyuan, in terms of nature, initially and even in quite a long period, was not a mainstream education mode with target of national examination-Keju (Note 3), but an institution with function of education. Later, Shuyuan was recognized officially, and consisted into education system in ancient China. Compared with official education institution, Chinese Shuyuan was featured with more freedom, though it's not much supported by the state financially; compared with private school, Chinese Shuyuan enjoyed much autonomy on its own administrative system and individual teaching and learning mission. On this perspective, Chinese Shuyuan was not a private school, targeting Keju only. Generally, Shuyuan was, on its nature, a special teaching institution, assisted by private parties and granted by official organizations (Ting, 2006).

\subsection{Features: Autonomy}

\subsubsection{Autonomy on Establishment of a Shuyuan}

Shuyuan was a very special education mode. Usually, opening a Shuyuan just relied on capacity and fame of founders who were masters recognized in certain fields and certain regions. Consequently, establishment of a Shuyuan demonstrated the founders' individual researching achievement, style and favor on pursuing knowledge. Due to such autonomy, founders of Shuyuan were able to lecture in their own places with academic topics they were initated and exploring; Learners got together to listen and inquire towards masters they've admired and respected; and founders of Shuyuan were dominant all aspects of Shuyuan: teaching and learning.

In other words, Shuyuan was an individual educational institution established by a master with his reputation of wisdom, aiming to transmit and pursue academic intelligences on his own favor.

\subsubsection{Autonomy on Management}

With development of Shuyuan, some lecturers working in their own Shuyuan finally were recommended into bigger Shuyuan founded by those more influential figures who were mostly prestigious both in their humble morality and scholarly wisdom, and sometimes by officials who were teaching in a Shuyuan part-time. A bigger Shuyuan meant there was a need of systematical management.

Bigger Shuyuan was able to draft and implement its rules and regulations, including punishing and praising items, which was mutual for both lecturers and learners. The most well known Shuyuan regulations were Learning Rules of White Deer Cave Shuyuan (白鹿洞书院) set up by scholar Zhu Xi; LiZe Shuyuan Learning Rules by scholar Lv Zuqian (Ying, 2006). With the rules and regulations, a systematical management was operated in Shuyuan instead of individual masters' preference. However, the style of Shuyuan was still determined by the dominant master-Shan Zhang (Note 4).

Shan Zhang is another tag for systematical management of Shuyuan. With more masters joining into one bigger Shuyuan, the founder of a Shuyuan, or the one among all masters in the Shuyuan with mostly great reputation turned into Shan Zhang who was dominant figure of Shuyuan. Besides, in the management, there were Vice Shan Zhang and teaching assistants who were cooperated to manage Shuyuan in every aspect. Sometimes, students were engaged into the management of Shuyuan, which could be considered the very first trial of involving learner into managing an educational institution.

From above depict, Chinese Shuyuan was able to be considered an ever pioneer in constructing its particular management of autonomy highlighting student-engagement in China history. 


\subsubsection{Autonomy on Academic Researching}

In Chinese history, scholars in mainstream were mostly disciples of Confucius, insisting in performance featured with Dao spirit, who preferred autonomy on lecturing and academic freedom. Since then, the concept of autonomy on learning and teaching had been effecting in academic circle and inherited generation by generation: autonomy on learning material and academic researching topics among scholars across Chinese territory in period of Warring States; autonomy on lectures given by scholars in Shuyuan in Han Dynasty (202 A.D.-220 A.D.); and autonomy manifested in Qingtan (清谈, Note 5) favored by great masters of Wei and Jin Dynasty (220 A.D.-420 A.D.), when Daoism was another booming philosophy and favored researching focus. Under such culture convention on academia, it's implicit that autonomy was ever the essence of scholarly intelligences and the favor pursued by all academics. Shuyuan completely echoed these values.

With convention of autonomy on pursuing academic intelligences, scholars, in Shuyuan, with different views were lecturing together, stressing on exchanging and discussing academically, freely of course. Lecturing (论道, Note 6), popular after Nan Song Dynasty (1127 A.D.-1279 A.D.), became a teaching and researching method in Shuyuan, through which teachers and learners joined together in academic argumentation and interpretation. In addition to other academic activities organized locally, Lecturing boosted Chinese Shuyuan into a regional educational and academic center. Shan Zhang and leading teachers with most talents decided the style of lectures of an individual Shuyuan and teaching content. Consequently, teaching contents were enriching, broadening with great flexibility and under autonomy.

In terms of autonomy, Chinese Shuyuan was able to organize its teaching and assessing procedure, emphasizing significant achievement on both morality and intelligence

\subsubsection{Autonomy on Learning}

Shuyuan, as mentioned before, was with purpose of inheriting and transmitting culture and intelligences through teaching and learning. Due to this goal, learners of Shuyuan were able to come and go to Shuyuan at anytime without concerning their different social status and no entrance examinations at all, where was considered just a place of pursuing knowledge and obtaining academic instruction, targeting teaching and learning for goodness of human. So, lecturers (teachers) and learners in Chinese Shuyuan preferred self-learning, independent exploring and researching, and the like through flexible curriculum which granted lecturers and learners great possibility to develop their own talents.

In other words, self learning, independent researching and teachers' as assistant to learners for challenging and inquiring topics they were favored etc, were the key activities in a lecture that was not designed for obtaining higher scores, but enhancing learners in morality and intelligences towards a higher level.

\subsubsection{Other Feature: Highlighting Construction of Humble Morality Through Teaching and Learning}

Learners and lecturers shared a warm atmosphere in Chinese Shuyuan, establishing a long-lasting tutor-learner relationship. In a whole, lecturers in Chinese Shuyuan were knowledgeable and expertise, with higher level morality and were devoted to teaching. Basing on high achievements in both morality and wisdom, lecturers were admired and respected by learners, who were good modals in every aspect for learning that attracted new learners to come for pursuing knowledge and expertise, morality and integrity of course continuously. This evidenced that loving learners and being respected by learners, such educational concept, highlighted in Chinese Shuyuan has always been being considered a conventional concept in Chinese education.

From above mentioned, autonomy was the very essence of Shuyuan, which is still paramount values pursued by modern education today. With autonomy, an active learner, humble person with life-long learning concept to pursuing knowledge for goodness of mankind was stressed as a very core in Shuyuan. In fact, it's just what educators in modern university prefer mostly and undergraduates are lack of under the current situation. On this aspect, it's supposed that the essence and spirit of autonomy in Shuyuan are the fundamental inspiration of those pioneer educators and key universities when they designed and implemented reforming project towards undergraduates, and entitle resident college with name after Shuyuan.

\subsection{Vanish of Chinese Shuyuan}

From Yuan Dynasty (1271 A.D.-1368 A.D.), there was a tendency and movements for rejuvenating official education institutions that were ruined due to politically unstable conditions of the state. In Ming Dynasty, some officials (Note 7) and Neo-Confucius scholars (Note 8) intended to make efforts to remedy official education institutions and secure Keju through development of Shuyuan. But, such campaign terrified Yandang Party (阉 党, Note 9), especially shocking its leader Wei, Zhongxian, because the newly established Shuyuan was progressed into political centers for scholars who pursuing political freedom, where political issues rather 
academic topic were discussed. Shuyuan was getting into a powerful political force. Among these Shuyuan, newly Donglin Shuyuan (东林书院) was a paramount one. Lectures and learners in this Shuyuan, as well as sponsors and supporters of the Shuyuan were called members of Donglin Party (东林党, Note 10). Terrified by Donglin Party's influential impact among scholars and politics, powerful eunuch Wei, Zhongxian ordered to kill them all. Most Shuyuan collapsed in this political campaign. Those survived Shuyuan all turned into serving Keju, functioning like official educational institution and private schools, giving away from criticizing political issues, and giving up autonomy the essence of Shuyuan ever claimed since its emerged and claimed its distinctive identity. Otherwise, these Shuyuan would be vanished soon. Responding to this transformation and the condition of Shuyuan, in the second year of Tianqi, Ming Dynasty (1622), Li, Yingshen who was charged of teaching in White Deer Cave Shuyuan, Jiangxi Province proposed that each Shuyuan should be granted quota of examinees on taking part into Keju, or Shuyuan would be allowed to recommend excellent learners to the Xiang Shi (乡试, Note 11), a most influential examination in Keju system. This proposal was called Dongxue Keju (洞 学科举, Note 12), by which Shuyuan finally was turn into a part of Keju system. Shuyuan was not a place for freely discussing academic topic or freely exchange political views, but one part of official education or one private school, serving Keju system which claimed function of screening officials for the state with higher scores. On this case, there was no Chinese Shuyuan any longer in terms of its distinctive feature-autonomy.

After Opium Wars, the entrance of the country was broken through by 'sharp canons and speedy warring boats' of foreign invaders. Newly schools dedicated for teaching and learning science and technology from western counties were established here and there in order to defend the motherland and fight 'against invading foreigners by learning their science and technology'. Qing government finally adopted proposal of Zhang, Zhidong and Liu, Kunyi (Note 13), and launched a project basing on the proposal: transforming Shuyuan in provincial level into universities, Shuyuan in city, middle schools, Shuyuan in county, elementary schools, besides, many kindergartens was built for learners before elementary schools. So far, Chinese Shuyuan vanished and new modern schooling was emerging.

\section{Chinese Residential College}

Bearing mind with those mentioned above, let's turn into the institution affiliated in Fudan University, or in Hongkong Chinese University and the like, which are named after Shuyuan in its Chinese translation while it's translated into English with residential college.

\subsection{Attributes of Residential College in Chinese University}

Like Fudan University, Xi' an Jiaotong University is another one pioneer to operate its residential colleges or named 'Shuyuan' Project. Pengkang Shuyuan (彭康书院) is first Shuyuan on the campus built up in 2006. Now, Xi' an Jiaotong University has developed 9 Shuyuan altogether so far. Stated on the website of Xi'an Jiaotong University, all these Shuyuan covering all undergraduates are aiming to "offer a platform to assist students to develop in all round with a series of educational activites..." (Pengkang, 2019), "(Shuyuan)commit to reach the target of enhancement of morality and transmit of Chinese ideology" (WenZhi, 2019). In Fudan University's homepage, Shuyuan is under management of Fudan College that is a newly rebuilt and covers the management of undergraduates affairs. There is, in Shuyuan of Fudan University, an individual administrative office-Office of Tutor that is specialized for management of Shuyuan tutors; working out tutor's routine activities; supporting comprehensively educational activities organized in Shuyuan; and supporting regular activities in Shuyuan and designing and implementing Shuyuan's developmental plan (Shuyuan life, 2019). Students in these Shuyuan usually are offered some activities more like something listed extra curriculum, not academic courses mostly (Students activities, 2019) Concerning on this routine activities, it's explicit that "This special institution is expected to promote students morality construction, train them with elegant disposition, good civilian quality and highly social responsibilities as well as social interaction capacity, leadership competence and concepts of science and diplomacy through various active teaching mode and strict management" (Reviewing and exploring, 2011).

Today, Shuyuan (书院) (residential college in English) on campus of Chinese university is a physically structured institution, administratively paralleling to all subordinating institutions on campus, charging with student affairs. Chinese residential college is dedicated to assist university students to construct their comprehensive literacy in all round, particularly enhancing and improving their ideology construction through "living in Shuyuan which is a key part of composing extra-curriculum and campus culture" (Renzhong, 2019) 


\subsection{Features of Chinese Residential College: Student Management System}

\subsubsection{Managing Students Through Their Living Places}

Residential colleges in Chinese universities are residential complex towards better management of students. Freshmen in those pioneer universities all need to be enrolled into one residential college, in line with certain rules like majors, countries and regions, races etc. where they are accommodated. Students living in one Chinese residential college are studying their majors in different academic institutions. Such mixing living management offers chances to broaden and enhance the exchanges between undergraduates majoring various disciplines. The first-year undergraduates must apply one year's course in Shuyuan, studying introductory education and comprehensive courses of art and science, then, they are allowed to learn their major courses in academic institutions. Such reform of Shuyuan changes the conventional student management that totally depends on academic institutions. It's reported that the biggest Chinese residential college in Xi'an JiaoTong University enrolls 3,4000 students, the smallest one, 600; FuDan University, as stated before, construct 5 Shuyuan that are different in its conceptions, but same in administration.

In short, Chinese residential college today is not only a living space, but another space for managing students except conventional management in each academic department.

\subsubsection{Managing Student Through 'Tutor + Assistant Teacher System'}

There are three types of teacher serving students in Xi'an Jiaotong University: residential teacher who help students to deal with routines and psychological problems; residential learning tutor who help students with their academic difficulties just in residence; and last type is part-time teacher who mainly are students. It's a position offered to students who wants to experience being a teacher, in order to promote the understanding between teachers and students, as well as to assist students' capacity in all round. In Fudan College, there are tutors and part-time tutors in its Shuyuan, who are responsible to tutor students in all aspects. Since 2011, Fudan College has initiated new plan in its several residential colleges. Conventionally, tutor and students ratio was 1:40, and the trial ones would be 1:8 aiming to perform a more personalized tutoring plan. Professor Lu, then vice president said, "the trial residential colleges would assist to cultivate students with self-managed competence with activity room, coffee bar, which would be operated by students themselves; students are encouraged to set up their own societies, academic ones and professional ones as well; the residential college would offer more opportunities to students to communicate internationally through actively exchanging activities with some Liberal Arts Colleges (Note 14) in United States, broadening international visions' (Zhang, 2011). Additionally, the Chinese residential colleges affiliated into same university usually work under each own developing plan, constitution and rules, presenting themselves with their own different flag, badge, motto, student union, league working committee, a set of management components.

4.2.3 Managing Students Through Liberal Art Education Course that Are Dedicated to Cultivate Humane Literacy Among Undergraduates

Residential colleges of HongKong Chinese University are responsible for courses of liberal art education, with purpose of promoting academic thinking and cultures through its various informal educational activities (Unique Shuyuan, 2019). Many conventions observed in Chinese residential college today are experiences learned from HongKong Chinese University where Shuyuan, the term came back and was first used on the Chinese translation of the complexes offered to undergraduates. In fact, HongKong Chinese University just learn such management from its counterparts in western countries where residential college has been developed well for its commitments towards higher education. For example, freshmen of Fudan University, as mentioned before, would learn in residential colleges in one academic year then turn to learn academic program courses in their academic institutions in second academic year. One purpose of this management in Fudan University is to promote humanistic literacy that would be helpful in their academic studying, employment and social living in long run. The residential college centers the goal of cultivating students' humanistic literacy through its various activities, which represents sort of connection between Shuyuan and Chinese residential college while it's the distinction between Chinese residential college and residential college in western countries.

From the analysis above, management is the key word able to depict the outline of Chinese residential college in a whole. Even there are some teaching tasks, like compulsory courses in liberal art education, it's implicit with purpose of managing students as whole.

\section{Comparison}

\subsection{Nature Differences}

Shuyuan (书院) in ancient China was an individual institution, not an official education institution, a private 
school neither. Chinese Shuyuan emerged with the purpose of culture inheriting and transmitting, academic freedom pursuing, with which it even had an impact into political issues in certain period basing on its highly academic advancement. In Chinese history, Chinese Shuyuan was a legacy in education. Jiang, Guangxue, ex-director of Youth Researching Center of Beijing University ever claimed, "Chinese Shuyuan, such unique education mode, was featured with Chinese culture and tradition. Lecturers and learners in Chinese Shuyuan were all keen on researching and transmitting classic culture; learners were educated to be a men with elegant disposition, capacity to take care of family as well as responsibility of engaging into states' affairs, mastery of knowledge of all kind to promote and protect his/their motherland in need, all of which are essentials in Chinese culture that have been passing down generations by generations, connoting spirits of being with strong will and integrity, perseverance and profoundness; benevolence and moderate" (Zhi yuan, 2011).

In terms of the nature stated above on Chinese Shuyuan, technically speaking, Chinese residential college is named after Shuyuan in Chinese, but it's only an administrative institution which managing students' affairs, a non-academic section on a modern university campus. It's not an individual institution but affiliated towards a university. As known well, modern university is keen on scientific achievement, which overwhelmingly emphasizes social satisfaction underlying conception of utilitarianism, rather committing culture transmitting and academic freedom that was taken the essence of Chinese Shuyuan in ancient time.

\subsection{Different Teaching Tasks}

Chinese residential colleges offer, in some cases, courses of liberal arts education with clear purpose of serving ideological construction and highlighting patriotism towards motherland. And such courses are compulsory, not optional for all freshmen. Such learning design is different from learning in academic departments and schools on the campus. For example, residential colleges in HongKong Chinese University, Fudan University and Xi' an Jiaotong University are administering a set of lectures and disciplinary instructions around liberal arts, aiming to assist students' ideological and political conception construction so as to manage students effectively, support students learning, assist interaction between teachers and students, with which learners are able to apply their academic programs in coming up second academic year. On such condition, Chinese residential college doesn't take charge of academic teaching and learning, neither researching. In fact, it is just a new home for university students who are living away from their parents at very first time.' Zhen, Nanning, then President of Xi'an Jiaotong University said, "Chinese residential college is, in a metaphor, playing a role of mother, while academic institution a father. Academic institutions support students to learn and research in their degree programs while residential colleges assist students to develop comprehensively in all rounds" (Jiang et al., 2011). On this metaphor, teaching tasks and goals in residential college on Chinese campus are quite different from that in other academic schools, let alone that in Shuyuan which targeted in culture researching and knowledge developing, pursuing academic freedom.

\subsection{Different Management System}

Chinese Shuyuan was run 'freely'. Chinese Shuyuan was built up with autonomy and managed freely with permission of the state that launched policy of 'Xuetian' (学田制, Note 15), a system financially guaranteed Shuyuan routine operation. It's considered that Shuyuan was the very first education mode that owned privately with publicly financial support, sometimes partly. When it comes to Chinese residential college today, it's found that it is just an administrative institution affiliated towards a university, serving the demands and needs of university. Staff of Chinese residential colleges is administrative staff serving university's administration tasks and demands, who are supervised and managed by university administrative system, paid by university of course.

In short, Shuyuan was an independently institution, privately own while residential college today is a section of a unit without autonomy in its management.

\subsection{Different Learners}

Learners in Chinese Shuyuan were pursuing learning and researching, because Chinese Shuyuan was not a public institution on its origin and not a private school, specifically, which was not set up to assist learner to take part into examination of all kind, around Keju mostly of course, but a place for exploring and discussing academic issues. On this aspect, learners in Shuyuan attempted to inherit culture and transmit culture, pursuing freedom of academy. Distinctively, students in Chinese residential colleges today have to select courses compulsorily because such courses in residential college are the prerequisite for their academic studying in coming up second academic year. Of cause, before enrolled into different residential colleges, students have to first pass national entrance examination towards universities and colleges, which were not requirement for learners who would like to listen to a master's lectures in Shuyuan hundreds of years ago. 


\section{Discussion and Analysis}

It's explicit that, basing on such statement and analysis, Shuyuan in ancient China and Chinese residential college are two different educational mechanisms in terms of its nature, feature and function. The very first motivation to establish Chinese residential college was to enhance or manage undergraduates' affairs efficiently, which is emerging from the need of management not academic related. Additionally, Chinese residential college attempts to survive the value of Shuyuan ever held and insisted, inheriting and transmitting brilliant literature achievements, culture achievements and the like in order to impact, even construct the correct vision and standard of being a lifelong learner, an active learner, and a person with awareness and capacity of socially interacting. But, Chinese residential college is a copy of residential college from foreign college in its physical complexes at very first design. Its intent no doubt is more inclining to the concept of resident college in foreign universities, where residential college is demonstration of management system in a modern university from its establishment.

On concerning the most distinction of Shuyuan and Chinese residential college: autonomy on teaching and learning, Shuyuan was recognized an initiative on education in Chinese history, even in the world, where was run with high autonomy, freely exploring new knowledge, and dedicated for human and his/her well-being in life long process. There is no doubt that Shuyuan, with such uniqueness on its commitment on culture transmit and knowledge explore, is booming inspiration for modern education worldwide and of course is considered a solution for Chinese educators and pioneers universities that concerning issues of Chinese higher education and Chinese undergraduates a lot. However, such design, with the establishment of residential colleges on Chinese campus, usually very good universities, like Fudan University, Xi'an Jiaotong University, Southern University of Science and Technology, named after Shuyuan, are not able to focus on academic lecturing with purpose of knowledge pursuit, culture transmit, because the nature of residential college determines its function and effects as well. The pioneer 'Shuyuan' projects (physically presented and operated in complexes of residential college) try to combine the essential concept of Shuyuan in ancient China and implementation of residential college well working in western universities in order to work out its way towards assisting undergraduates under tough circumstances they are confronting today.

\section{Conclusion}

Undergraduates are highlighted in China today, and Chinese higher education institution are facing unprecedentedly challenges coming at home and abroad, so assistance of undergraduates in every aspect, especially the construction of ideology and enhancement of Chinese excellent culture awareness and implementation in the fast globalization development processing is the very important task and foremost concerns in China. Shuyuan as an educational mode, with its unique features actively going on with other education modes in Chinese history, ever presented its uniqueness and function with autonomy that was a very initiative and advanced concept on education, not just in China, but the whole world. Insisting on such advancement of Shuyuan, Chinese residential college is constructed in universities of Chinese mainland basing on such concern. No matter what Chinese residential college is defined and practiced in those pioneer Chinese universities, it's explicit that Chinese residential college is an effort in terms of its goal and design, but not an effort available among Chinese higher education institution and the efficiency of the project hasn't been inspected and computation. However, Shuyuan, its essence of pursuit of autonomy on teaching and learning, targeting knowledge exploring and culture transmitting, comes back into the vision of education that would greatly help educators and stakeholders of the field to figure out better plan towards education issues in China and the whole world today.

\section{Reference}

Fudan College. (2019). Students' activities. Retrieved September 10, 2019, from http://www.fdcollege.fudan.edu.cn/xssh/list.htm

Fudan University. (2019a). Renzhong Shuyuan. Retrieved September 10, 2019, from http://www.renzhong.fudan.edu.cn/gk/list.htm

Fudan University. (2019b). Shuyuan Life. Retrieved Sepember 10, 2019, from http://www.fudan.edu.cn/2016/channels/view/116/

Fudan University. (2019c). Tutors' Office in Shuyuan. Retrieved September 10, 2019, from http://www.fdcollege.fudan.edu.cn/9435/list.htm

Fudan University. (2019d). The Residential College System. Retrieved September 10, 2019, from https://rc.um.edu.mo/the-residential-college-system- $\%$ e6\%9b\%b8\%e9\%99\%a2\%e7\%b3\%bb\%e $7 \%$ b5\%b1/ 
HongKong Chinese University. (2019). Unique Shuyuan Policy. Retrieved September 10, 2019, from http://www.cuhk.edu.hk/chinese/college/system.html

Huang, Y. Z. (1894). Jingji These. Jinagshu, Nanjingjiangshe Press. China. Quoted from Zhu, H. M. (2010). Autonomy in Chinese Shuyuan. University Education Sicence, 3, 73-78.

Jiang, H. B., Yang, Y., \& Ying, S. C. (2011, September 16). What happened after reform of Shuyuan establishment? ChinaDaily. Retrieved from http://culture.people.com.cn/GB/15671860.html

Oversea Colum, China Daily. (2011, October 11). Reviewing and exploring Shuyun culture, Fudan University attempting a connection between tradition and modern education. Received from https://www.chsi.com.cn/jyzx/201110/20111011/238775736.html

Ryan, M. B. (2001). A Collegiate Way of Living.Residential Colleges and A Yale Education. Jonathan Edwards College, Yale University.

sina.com. (2005, October 5). On social and culture function of Chinese Shuyuan. Retrieved from http://news.sina.com.cn/c/2005-10-05/06227097800s.shtml

$\mathrm{Su}$, Y. B. (2010). Talents Cultivation Mode through Shuyuan System in Xi'an Jiaotong University: The Implementation and Exploration. In S. D. Xiong et al. (Eds.), Liberal Art Education and Higher Education: Exploration in China (p. 129). Beijing: Science Press.

Ting, Y. (2006, Nov. 22). Chinese Shuyuan Policy. Retrieved from http://www.confucianism.com.cn/jiaoyu/Show.asp?id=10977

University of Macau, Chao Kuang Piu College. (2019). What is Residential College? Retrieved September 10, 2019, from https://ckpc.rc.um.edu.mo/

Xi'an Jiaotong University. (2019a). Pengkang Shuyuan. Retrieved September 10, 2019, from http://www.xjtu.edu.cn/bksy/pksy.htm

Xi'an Jiaotong University. (2019b). Wenzhi Shuyuan. Retrieved September 10, 2019, from http://www.xjtu.edu.cn/bksy/wzsy.htm

Zhang, Q. Q. (2011, Aug 22). Vice President of Fudan University: trial of establishment of 4-year Shuyuan institution, where tutors and students rate would be 1:8. Retrieved from http://news.ifeng.com/mainland/detail_2011_08/22/8586102_0.shtml

\section{Notes}

Note 1. Residential college, or residential hall in some cases, is known and developed well in Western universities, the most successful case is residential college in Yale University. On this paper, in order to make identification on terms used, residential college is referred to the mode in Western universities, and Chinese residential college is affiliated to Chinese university, which is name after Shuyuan (书院) in its Chinese terms.

Note 2. White Deer Cave Shuyuan (白鹿洞书院) was built during 1th year of Shengyuan of Nantang Dynasty (940 B.C.). In Song Dynasty (960 B.C.-1279 B.C.), well known Scholar Zhu Xi was commissioned the local official at today Lushan City, Jiangxi province. He rebuilt the Shuyuan (书院) and lectured over there himself. Attributed to Zhu Xi, Whilte Deer Cave became a most influential Shuyuan (书院) that was recognized a birthplace for Chinese education, one importance educational institution since the late Song Dynasty to early Qin Dynasty.

Whit Deer Cave Shuyuan is under state protection after formation of new China, and is recognized one of 4 most influential Shuyuan, ranking No.1. Other 3 are Yuelu Shuyuan (岳麓书院), Yingtian Shuyuan (应天书院), and Shigu Shuyuan (石鼓书院). In 1988, White Deer Cave Shuyuan was recorded as an important institution protected by the state.

Note 3. Keju (科举) was a national examination system, holding nationally to all who were capable of being a scholar, and most importantly being future officials of the country. It covered a serial of examinations in a hierarchical row. Only those passed the first level examination would be qualified to the examination in next level It's concerned a fair system to select the most capable person for the governance of a state. The modern national examination in China is evolved from Keju system.

Note 4. Shan Zhang (山长) referred to a scholar with great reputation, head of other lectures in a Shuyuan, sometimes just the founder of Shuyuan. 
Note 5. Qingtan (清谈) was a particular discourse preferred in period of Wei and Jin Dynasty (220 A.D.-420 A.D.) when the state was under separation and chaos spread over the country. Qingtan, in this period, presented two types: politically discussing empirical issues in early stage; and apart away political issues in late stage.

Note 6. Lecturing, like academic seminar today, was initiated in Song Dynasty and it contributed to disseminate and develop academic views and establish a learning and researching atmosphere as well as boosted Chinese Shuyuan in quit a period. The event was featured with exploration, argumentation, and analysis on a dispute existing among different schools, as well as transmitting a new perception or conception favored by scholars.

Note 7. Shidafu (士大夫) was a group of people with special social status that was evolved from official system in Zhou Dynasty (1046 A.D.-256 A.D.), including state officials, noble families, higher ranking soldiers. They enjoyed most privilege in every aspect of social life. This class was ranked No.1 among Chinese social class system: Shi (Shidafu), Nong (farmer and tenant), Gong (technician), Shang (businessman).

Note 8. Neo-Confucius scholars were in favor of newly Confucius philosophy that was developed in Song Dynasty and Ming Dynasty with the impact of philosophy of Buddhism and Taoism.

Note 9. Yandang Party (阉党) was a special political party in Chinese history, booming in Ming Dynasty (1368-1644) consisting of officials and eunuchs with power. Eunuchs engaging into state governance was a peculiar phenomenon in Chinese history, leading to social chaos and crisis in several Chinese historical stages. The most case happened in the end of Ming Dynasty when Emperor Tianqi was on the throne (1605-1627). Wei, Zhongxian, then, a Eunuch and a political power ever since, led Yandang Party to reach an unprecedentedly political status, which produced national chaos and panic.

Note 10. Donglin Party (东林党) referred to a party composed of a group member who were ever lecturers and learners in new Donglin Shuyuan (新东林书院) (1604-1625.) that was rebuilt by Gu, Xiancheng (1550-1612) and other sponsors on old Donglin Shuyuan (旧东林书院) (1111-1129) established by Yang, Shi (1053-1135) in Song Dynasty. Due to political disputes with Yangdang Party led by eunuch Wei, Zhongxian, Donglin Party was slaughtered over by Yandang Party.

Note 11. Xiangshi (乡试), Examination in provincial level, was considered a most influential examination in Keju system and a most competitive one, because the number of examinees were restrained with quota granted to the province where examinees lived that caused greatly completion for the quality of being examinees, and Xiangshi held once in three years in each province at the same time, when the examination was a gala of all social classes.

Note 12. Dongxue Keju (洞学科举, 1622) was a transformational proposal for Shuyuan under the depression condition. It's proposed that learners of Shuyuan could be granted quota of taking into Xiangshi, a very influential examination in Keju system.

Note 13. Zhang, Zhidong (1837-1909) and Liu, Kunyi (1830-1902) were both high ranking officials in state court, and influential figures appealing reform nationwide. They proposed a motion of reform three times, of which establishment of new schools, reform of governance and learning from foreign technology were the main sections. Additionally, Zhang, Zhidong was entitled with 'four outstanding officials in late Qin Dynasty' with other 3 officials: Zeng, Guofan, Li, Hongzhang, Zuo, Zhongtang.

Note 14. A liberal arts college or liberal arts institution of higher education is a college with an emphasis on undergraduate study in the liberal arts and sciences. Such colleges aim to impart a broad general knowledge and develop general intellectual capacities, in contrast to a professional, vocational, or technical curriculum.

Note 15. Xuetian policy (学田制) was a state policy. With this policy, Shuyuan was allowed to rent the land, some from Government, some just owned by Shuyuan, to tenants nearby and got rents for its operation. Xuetian policy financially supported the operation of education institutions, including Shuyuan. In fact, there wasn't any national policy for maintaining education institution before Song Dynasty (960-1279). After Emperor RenZong (1022-1063) in Song Dynasty, there was a fund-raising system for education, of which Xuetian policy was very important, integrated by other systems and policies.

\section{Copyrights}

Copyright for this article is retained by the author, with first publication rights granted to the journal.

This is an open-access article distributed under the terms and conditions of the Creative Commons Attribution license (http://creativecommons.org/licenses/by/4.0/). 